\title{
A New Scheme for Discrete HJB Equations
}

\section{Zhanyong Zou}

School of Mathematics and Statistics, Guangdong University of Finance \& Economics, Guangzhou, China

Email: yong china@126.com

Received 2 August 2014; revised 28 August 2014; accepted 10 September 2014

Copyright (C) 2014 by author and Scientific Research Publishing Inc.

This work is licensed under the Creative Commons Attribution International License (CC BY).

http://creativecommons.org/licenses/by/4.0/

c) (7) Open Access

\section{Abstract \\ In this paper we propose a relaxation scheme for solving discrete HJB equations based on scheme II [1] of Lions and Mercier. The convergence of the new scheme has been established. Numerical example shows that the scheme is efficient.}

\section{Keywords}

\section{Iterative Algorithm, Relaxation Scheme, HJB Equation, Convergence, Existence}

\section{Introduction}

Consider the following Hamilton-Jacobi-Bellman (HJB) equation:

$$
\begin{array}{ll}
\max _{1 \leq i \leq k}\left\{L^{i} u-f^{i}\right\}=0 & \text { in } \Omega, \\
u=0 & \text { on } \partial \Omega,
\end{array}
$$

where $\Omega$ is a bounded domain in $R^{d}, L^{i}, i=1, \cdots, k$, are elliptic operators of second order. Equation (1.1) is arising in stochastic control problems. See [2] and the references therein.

Equation (1.1) can be discretized by finite difference method or finite element method. See [1] [3] and the references therein. Then we obtain the following discrete HJB equation:

$$
\max _{1 \leq i \leq k}\left\{A^{j} U-F^{j}\right\}=0,
$$

where $A^{j} \in R^{n \times n}, F^{j} \in R^{n}, j=1, \cdots, k$. Equation (1.2) is a system of nonsmooth nonlinear equations. Many numerical algorithms for solving (1.2) have been proposed. See [4]-[12] and the references therein.

[1] has given two iterative algorithms for solving (1.2). At each iteration, a linear complementarity subproblem or a linear equation system subproblem is solved. See also [4].

Scheme I.

Step 1: Given $\varepsilon>0, m:=1$, for some $j$ we find $U^{0, k}$ such that 


$$
A^{j} U^{0, k}=F^{j} .
$$

Step 2: Let $N=(m-1) k, U^{N, 0}=U^{N, k}$. For $j=1, \cdots, k$, we find $U^{N+j, j}$ such that

$$
\max \left\{A^{j} U^{N+j, j}-F^{j}, U^{N+j, j}-U^{N+j-1, j-1}\right\}=0 .
$$

Step 3: If $\left\|U^{m k, k}-U^{N, 0}\right\|<\varepsilon$, then the output is $U^{m k, k}$, otherwise $m=: m+1$ and it goes to Step 2.

Assume $A^{j}=\left(a_{l s}^{j}\right), F^{j}=\left(F_{l}^{j}\right)$. Let

$$
A\left(p_{1}, \cdots, p_{n}\right)=\left(a_{l s}^{p_{l}}\right), F\left(p_{1}, \cdots, p_{n}\right)=\left(F_{l}^{p_{l}}\right) .
$$

That is: the lth row of matrix $A\left(p_{1}, \cdots, p_{n}\right)$ is the lth row of matrix $A^{p_{l}}$; the lth component of vector $F\left(p_{1}, \cdots, p_{n}\right)$ is the lth component of vector $F^{p_{l}}$. Now we formulate Scheme II of Lions and Mercier in the notation above.

Scheme II.

Step 1: $m:=0$, for some $j$ we find $U^{0}$ such that

$$
A^{j} U^{0}=F^{j} .
$$

Step 2: For $l=1, \cdots, n$, we find $p_{l}^{m}$ such that

$$
p_{l}^{m}=\min \left\{j \in\{1, \cdots, k\}:\left(A^{j} U^{m}-F^{j}\right)_{l}\right\}=\max _{1 \leq j \leq k}\left\{\left(A^{j} U^{m}-F^{j}\right)_{l}\right\} .
$$

Step 3: Compute $U^{m+1}$ as the solution of

$$
A\left(p_{1}^{m}, \cdots, p_{n}^{m}\right) U^{m+1}=F\left(p_{1}^{m}, \cdots, p_{n}^{m}\right) .
$$

Step 4: If $U^{m+1}=U^{m}$ then the output is $U^{m}$, otherwise $m=: m+1$ and it goes to Step 2 .

In the last decade many numerical schemes have been given for solving (1.2). But the above schemes are still playing a very important role. See [4]-[6] and the references therein.

In this paper we propose, based on Scheme II above, a relaxation scheme with a parameter $\omega$, which for $\omega=1$ is just Scheme II. In our numerical example, the new scheme with $\omega=0.8,0.9$ is faster than Scheme II $(\omega=1)$. The monotone convergence of the new scheme has been proved.

\section{New Scheme and Convergence}

We propose a new scheme which is an extension of Scheme II.

New Scheme II.

Step 1: Given $\varepsilon>0, \omega \in(0,1] \quad m:=0$, for some $j$ find $U^{0}$ such that

$$
A^{j} U^{0}=F^{j} .
$$

Step 2: For $l=1, \cdots, n$, find $p_{l}^{m}$ such that

$$
p_{l}^{m}=\min \left\{j \in\{1, \cdots, k\}:\left(A^{j} U^{m}-F^{j}\right)_{l}\right\}=\max _{1 \leq j \leq k}\left\{\left(A^{j} U^{m}-F^{j}\right)_{l}\right\} .
$$

Step 3: Compute $V^{m+1}$ as the solution of

$$
A\left(p_{1}^{m}, \cdots, p_{n}^{m}\right) V^{m+1}=F\left(p_{1}^{m}, \cdots, p_{n}^{m}\right) .
$$

Step 4: Compute

$$
U^{m+1}=(1-\omega) U^{m}+\omega V^{m+1} .
$$

Step 5: If $\left\|U^{m+1}-U^{m}\right\|<\varepsilon$ then output $U^{m}$ otherwise $m=: m+1$ and go to Step 2.

In [13] we proposed the following conditions for (1.2).

Condition $A^{*}$ All the matrices $A\left(p_{1}, \cdots, p_{n}\right), p_{l}=1, \cdots, m, l=1, \cdots, n$, are $M$-matrices. 
In [13] we have proved the following theorem.

Theorem 2.1 If Condition $A^{*}$ holds then (1.2) has a unique solution.

We have the following convergence theorem.

Theorem 2.2 Assume that Condition $A^{*}$ holds, and that $U^{m}, m=0,1,2, \cdots$ are produced by New Scheme II. Then $U^{m}$ is monotonely decreasing and convergent to the solution of (1.2).

Proof Since all $A\left(p_{1}, \cdots, p_{n}\right), p_{l}=1, \cdots, k, l=1, \cdots, n$, are $M$-matrices, $U^{m}, m=0,1, \cdots$ in New Scheme II are well defined.

First, we prove $U^{m}$ is decreasing monotonically, i.e.,

$$
\cdots \leq U^{m+1} \leq U^{m} \leq \cdots \leq U^{1} \leq U^{0} .
$$

By (2.3) we have

$$
A\left(p_{1}^{0}, \cdots, p_{n}^{0}\right) V^{1}=F\left(p_{1}^{0}, \cdots, p_{n}^{0}\right),
$$

which combining with (2.1) and (2.2) yields

$$
\begin{aligned}
A\left(p_{1}^{0}, \cdots, p_{n}^{0}\right) U^{0}-F\left(p_{1}^{0}, \cdots, p_{n}^{0}\right) & \geq A^{j} U^{0}-F^{j}=0 \\
& =A\left(p_{1}^{0}, \cdots, p_{n}^{0}\right) V^{1}-F\left(p_{1}^{0}, \cdots, p_{n}^{0}\right) .
\end{aligned}
$$

Since $A\left(p_{1}^{0}, \cdots, p_{n}^{0}\right)$ are $M$-matrices, (2.7) means

$$
V^{1} \leq U^{0} .
$$

By (2.4) we obtain

$$
U^{1}=(1-\omega) U^{0}+\omega V^{1}
$$

By $\omega \in(0,1]$, (2.8) and (2.9) we know

$$
U^{1} \leq U^{0},
$$

and

$$
V^{1} \leq U^{1},
$$

which and (2.10) implies

$$
V^{1} \leq U^{1} \leq U^{0}
$$

Similarly, by (2.3) we derive

$$
A\left(p_{1}^{1}, \cdots, p_{n}^{1}\right) V^{2}=F\left(p_{1}^{1}, \cdots, p_{n}^{1}\right),
$$

which combining with (2.2) and (2.6) implies

$$
\begin{aligned}
A\left(p_{1}^{1}, \cdots, p_{n}^{1}\right) V^{1}-F\left(p_{1}^{1}, \cdots, p_{n}^{1}\right) & \geq A\left(p_{1}^{0}, \cdots, p_{n}^{0}\right) V^{1}-F\left(p_{1}^{0}, \cdots, p_{n}^{0}\right) \\
& =A\left(p_{1}^{1}, \cdots, p_{n}^{1}\right) V^{2}-F\left(p_{1}^{1}, \cdots, p_{n}^{1}\right) .
\end{aligned}
$$

Hence we have

$$
V^{2} \leq V^{1}
$$

By (2.4), we have

$$
U^{2}=(1-\omega) U^{1}+\omega V^{2} .
$$

By (2.12), (2.13) and $\omega \in(0,1]$, we know

$$
U^{2} \leq(1-\omega) U^{1}+\omega V^{1},
$$


which combining with $\omega \in(0,1]$ and (2.11) we derive

$$
U^{2} \leq U^{1} .
$$

By (2.11), (2.12) and (2.13), we get

$$
V^{2} \leq U^{2}
$$

which combining with (2.15) implies

$$
V^{2} \leq U^{2} \leq U^{1}
$$

It is easy to derive by induction that

$$
V^{m+1} \leq U^{m+1} \leq U^{m}, m=0,1, \cdots,
$$

and

$$
V^{m+1} \leq V^{m}, m=0,1, \cdots
$$

It follows that (2.5) holds.

It follows from (2.2) and (2.3) that

$$
\begin{aligned}
\max _{1 \leq j \leq k}\left\{A^{j} V^{m}-F^{j}\right\} & =A\left(p_{1}^{m}, \cdots, p_{n}^{m}\right) V^{m}-F\left(p_{1}^{m}, \cdots, p_{n}^{m}\right) \\
& =A\left(p_{1}^{m}, \cdots, p_{n}^{m}\right)\left(V^{m}-V^{m+1}\right), \quad m=0,1, \cdots .
\end{aligned}
$$

Since the set $\left\{\left(p_{1}, \cdots, p_{n}\right): p_{l}=1, \cdots, k, l=1, \cdots, n\right\}$ is a finite set there exist positive integers $q$ and $m$ with $q>k$ such that

$$
\left(p_{1}^{q}, \cdots, p_{n}^{q}\right)=\left(p_{1}^{m}, \cdots, p_{n}^{m}\right)
$$

Therefore, we have

$$
\begin{aligned}
& A\left(p_{1}^{q}, \cdots, p_{n}^{q}\right)=A\left(p_{1}^{m}, \cdots, p_{n}^{m}\right), \\
& F\left(p_{1}^{q}, \cdots, p_{n}^{q}\right)=F\left(p_{1}^{m}, \cdots, p_{n}^{m}\right) .
\end{aligned}
$$

Then by (2.2) we obtain

$$
V^{q+1}=V^{m+1},
$$

which and (2.17) results in

$$
V^{q+1}=V^{q}=\cdots=V^{m+2}=V^{m+1} .
$$

From (2.4), (2.16) and (2.19) we have

$$
U^{q+1}=U^{q}=\cdots=U^{m+2}=U^{m+1} .
$$

It follows from (2.18), (2.19) and (2.20) that

$$
\max _{1 \leq j \leq k}\left\{A^{j} U^{m+1}-F^{j}\right\}=0,
$$

which means $U^{m+1}$ is a solution of (1.2). The existence of solution has been proved.

Finally, we prove the uniqueness of solution. Assume $U$ and $U^{*}$ are solutions of (1.2), i.e.,

$$
\begin{aligned}
& \max _{1 \leq j \leq k}\left\{A^{j} U-F^{j}\right\}=0, \\
& \max _{1 \leq j \leq k}\left\{A^{j} U^{*}-F^{j}\right\}=0 .
\end{aligned}
$$


It is easy to see from (2.21) and (2.22) that there exist $\left(p_{1}, \cdots, p_{n}\right)$ and $\left(p_{1}^{*}, \cdots, p_{n}^{*}\right)$ such that

$$
\begin{aligned}
& A\left(p_{1}, \cdots, p_{n}\right) U-F\left(p_{1}, \cdots, p_{n}\right)=0, \\
& A\left(p_{1}^{*}, \cdots, p_{n}^{*}\right) U^{*}-F\left(p_{1}^{*}, \cdots, p_{n}^{*}\right)=0, \\
& A\left(p_{1}^{*}, \cdots, p_{n}^{*}\right) U-F\left(p_{1}^{*}, \cdots, p_{n}^{*}\right) \leq 0, \\
& A\left(p_{1}, \cdots, p_{n}\right) U^{*}-F\left(p_{1}, \cdots, p_{n}\right) \leq 0 .
\end{aligned}
$$

(2.23) and (2.26) implie $U^{*} \leq U$. But (2.24) and (2.25) implies $U^{*} \geq U$. Hence $U^{*}=U$. The proof is complete.

\section{Numerical Example}

We use example 2 in [4], i.e., $k=n=2, \Omega=(0,1) \times(0,1)$.

$$
\begin{array}{ll}
\max _{1 \leq i \leq 2}\left\{L^{i} u-f^{i}\right\}=0 & \text { in } \Omega, \\
u=0 & \text { on } \partial \Omega,
\end{array}
$$

where $\Omega=\{(x, y): 0<x, y<1\}$,

$$
\begin{gathered}
L^{1}=-(x+6)^{2} \frac{\partial^{2}}{\partial x^{2}}-(x+6)(y+2) \frac{\partial^{2}}{\partial x \partial y}-(y+2)^{2} \frac{\partial^{2}}{\partial y^{2}} \\
+[0.5(x+6)-4] \frac{\partial}{\partial x}+0.5(y+2) \frac{\partial}{\partial y}+1, \\
L^{2}=-(x+6)^{2} \frac{\partial^{2}}{\partial x^{2}}-0.8(x+6)(y+2) \frac{\partial^{2}}{\partial x \partial y}-0.75(y+2)^{2} \frac{\partial^{2}}{\partial y^{2}} \\
+[(x+6)-2] \frac{\partial}{\partial x}+(y+2) \frac{\partial}{\partial y}+4, \\
u=x(1-x) y(1-y), \\
f^{1}=f^{2}=\max \left(L^{1} u, L^{2} u\right) .
\end{gathered}
$$

The discretization of the above second order derivatives are:

$$
\begin{gathered}
\frac{\partial^{2}}{\partial x^{2}} \approx h^{-2} D_{h, x}^{+} D_{h, x}^{-}, \quad \frac{\partial^{2}}{\partial y^{2}} \approx h^{-2} D_{h, y}^{+} D_{h, y}^{-}, \\
\frac{\partial^{2}}{\partial x \partial y} \approx \frac{1}{2} h^{-2}\left[D_{h, x}^{+} D_{h, y}^{+}+D_{h, x}^{-} D_{h, y}^{-}\right],
\end{gathered}
$$

where $D_{h, x}^{ \pm}, D_{h, y}^{ \pm}$denote the forward and backward difference respectively in $x$ and $y, h=1 / 10, h=1 / 20$. We use New Scheme II to solve the discrete problem. Take $\varepsilon=10^{-5}, \omega=0.1,0.5,0.8,0.9,1.0$ and 1.1, 1.3, 1.5, 1.8, 1.9 respectively.

Table 1 and Table 2 show the $\infty$-norm of the residual $R=\max _{1 \leq j \leq k}\left\{A^{j} U^{m}-F^{j}\right\}$ when iteration terminates.

We see that $R \approx 0$ for $\omega \leq 1$ and $R$ is big for $\omega \in(1,2)$.

Table 3 shows the relation between iteration number $m$ and relaxation number $\omega(\omega \in(0,1])$. Table 4 and Table 5 show the value of $U^{m}$ at $(x, y)^{\mathrm{T}}=(0.5,0.5)^{\mathrm{T}}$ for $h=1 / 10$ and $h=1 / 20$ respectively.

We can see from Table 3 that the algorithm for $\omega=0.8,0.9$ is faster than that for $\omega=1$. Table 4 and Table 5 display the monotonicity of the algorithm. 
Table 1. $\infty$-norm of the residual $R$.

\begin{tabular}{cccccc}
\hline$\omega$ & 0.1 & 0.5 & 0.8 & 0.9 & 1.0 \\
\hline$\|R\|_{\infty}$ & & & & & \\
$h=1 / 10$ & $3.419 \mathrm{e}-004$ & $2.099 \mathrm{e}-011$ & $9.464 \mathrm{e}-012$ & $6.861 \mathrm{e}-012$ & $6.651 \mathrm{e}-012$ \\
$h=1 / 20$ & $6.630 \mathrm{e}-003$ & $1.784 \mathrm{e}-008$ & $6.653 \mathrm{e}-011$ & $6.062 \mathrm{e}-011$ & $8.169 \mathrm{e}-006$ \\
\hline
\end{tabular}

Table 2. $\infty$-norm of the residual $R$.

\begin{tabular}{cccccc}
\hline$\omega$ & 1.1 & 1.3 & 1.5 & 1.8 & 1.9 \\
\hline$\|R\|_{\infty}$ & & & & & \\
$h=1 / 10$ & $3.440 \mathrm{e}-000$ & $2.314 \mathrm{e}+001$ & $4.670 \mathrm{e}+001$ & $8.421 \mathrm{e}+001$ & $9.730 \mathrm{e}-000$ \\
$h=1 / 20$ & $1.667 \mathrm{e}-003$ & $4.323 \mathrm{e}+001$ & $1.754 \mathrm{e}+002$ & $4.323 \mathrm{e}+001$ & $2.089 \mathrm{e}+002$ \\
\hline
\end{tabular}

Table 3. Iteration number $m$.

\begin{tabular}{cccccc}
\hline$\omega$ & 0.1 & 0.5 & 0.8 & 0.9 & 1.0 \\
\hline$m$ & & & & & \\
$h=1 / 10$ & 200 & 198 & 107 & 90 & 124 \\
$h=1 / 20$ & 600 & 495 & 282 & 258 & 400 \\
\hline
\end{tabular}

Table 4. The value of $U^{m}$ at $(x, y)^{\mathrm{T}}=(0.5,0.5)^{\mathrm{T}}$.

\begin{tabular}{|c|c|c|c|c|c|}
\hline$\omega$ & 0.1 & 0.5 & 0.8 & 0.9 & 1.0 \\
\hline \multicolumn{6}{|l|}{$h=1 / 10$} \\
\hline$m=1$ & 1.091409800 & 1.086033962 & 1.082002083 & 1.080658123 & 1.079314164 \\
\hline$m=2$ & 1.089751377 & 1.080022728 & 1.074891194 & 1.073533844 & 1.076283661 \\
\hline$m=3$ & 1.088256293 & 1.075449958 & 1.072050161 & 1.071072814 & 1.073086733 \\
\hline$m=4$ & 1.086758364 & 1.073060086 & 1.069451302 & 1.068586924 & 1.072407806 \\
\hline Last $m$ & 1.065963994 & 1.065887109 & 1.065887109 & 1.065887109 & 1.065887109 \\
\hline \multicolumn{6}{|c|}{ Table 5. The value of $U^{m}$ at $(x, y)^{\mathrm{T}}=(0.5,0.5)^{\mathrm{T}}$. } \\
\hline$\omega$ & 0.1 & 0.5 & 0.8 & 0.9 & 1.0 \\
\hline \multicolumn{6}{|l|}{$h=1 / 20$} \\
\hline$m=1$ & 1.077654026 & 1.073664734 & 1.070672766 & 1.069675443 & 1.068678121 \\
\hline$m=2$ & 1.076493553 & 1.069008305 & 1.065427282 & 1.065027950 & 1.068036835 \\
\hline$m=3$ & 1.075236529 & 1.065915940 & 1.063091196 & 1.062134520 & 1.066011200 \\
\hline$m=4$ & 1.073996351 & 1.063479656 & 1.060857772 & 1.060476760 & 1.065563176 \\
\hline Last $m$ & 1.054467308 & 1.054409847 & 1.054409847 & 1.054409847 & 1.054409847 \\
\hline
\end{tabular}

\section{Funding}

This work was supported by Educational Commission of Guangdong Province, China (No. 2012LYM-0066) 
and the National Social Science Foundation of China (No. 14CJL016).

\section{References}

[1] Lions, P.L. and Mercier, B. (1980) Approximation numerique des equations de Hamilton-Jacobi-Bellman. RAIRO Numerical Analysis, 14, 369-393.

[2] Bensoussan, A. and Lions, J.L. (1982) Applications of Variational Inequalities in Stochastic Control. North-Holland, Amsterdam.

[3] Boulbrachene, M. and Haiour, M. (2001) The Finite Element Approximation of Hamilton-Jacobi-Bellman Equations. Computers \& Mathematics with Applications, 14, 993-1007. http://dx.doi.org/10.1016/S0898-1221(00)00334-5

[4] Hoppe, R.H.W. (1986) Multigrid Methods for Hamilton-Jacobi-Belman Equations. Numerische Mathematik, 49, 239254. http://dx.doi.org/10.1007/BF01389627

[5] Huang, C.S., Wang, S. and Teo, K.S. (2004) On Application of an Alternating Direction Method to HJB Equations. Journal of Computational and Applied Mathematics, 166, 153-166. http://dx.doi.org/10.1016/j.cam.2003.09.031

[6] Sun, M. (1993) Domain Decomposition Method for Solving HJB Equations. Numerical Functional Analysis and Optimization, 14, 145-166. http://dx.doi.org/10.1080/01630569308816513

[7] Sun, M. (1996) Alternating Direction Algorithms for Solving HJB Equations. Applied Mathematics and Optimization, 34, 267-277. http://dx.doi.org/10.1007/BF01182626

[8] Young, D. (1971) Iterative Solution of Large Linear Systems. AP, New York.

[9] Zhou, S.Z. and Chen, G.H. (2005) A Monotone Iterative Algorithm for a Discrete HJB Equation. Mathematica Applicata, 18, 639-643. (in Chinese)

[10] Zhou, S.Z. and Zhan, W.P. (2003) A New Domain Decomposition Method for an HJB Equation. Journal of Computational and Applied Mathematics, 159, 195-204. http://dx.doi.org/10.1016/S0377-0427(03)00554-5

[11] Zhou, S.Z. and Zou, Z.Y. (2008) An Itetative Algorithm for a Quasivariational Inequality System Related to HJB Equation. Journal of Computational and Applied Mathematics, 219, 1-8. http://dx.doi.org/10.1016/j.cam.2007.07.013

[12] Zhou, S.Z. and Zou, Z.Y. (2008) A New Iterative Method for Discrete HJB Equations. Numerische Mathematik, 111, 159-167. http://dx.doi.org/10.1007/s00211-008-0166-6

[13] Zhou, S.Z. and Zou, Z.Y. (2007) A Relaxation Scheme for Hamilton-Jacobi-Bellman Equations. Applied Mathematics and Computation, 186, 806-813. http://dx.doi.org/10.1016/j.amc.2006.08.025 
Scientific Research Publishing (SCIRP) is one of the largest Open Access journal publishers. It is currently publishing more than 200 open access, online, peer-reviewed journals covering a wide range of academic disciplines. SCIRP serves the worldwide academic communities and contributes to the progress and application of science with its publication.

Other selected journals from SCIRP are listed as below. Submit your manuscript to us via either submit@scirp.org or Online Submission Portal.
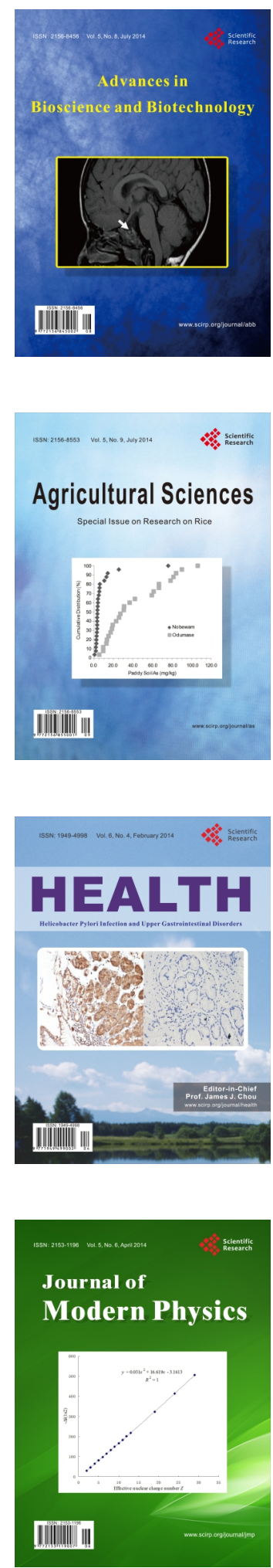
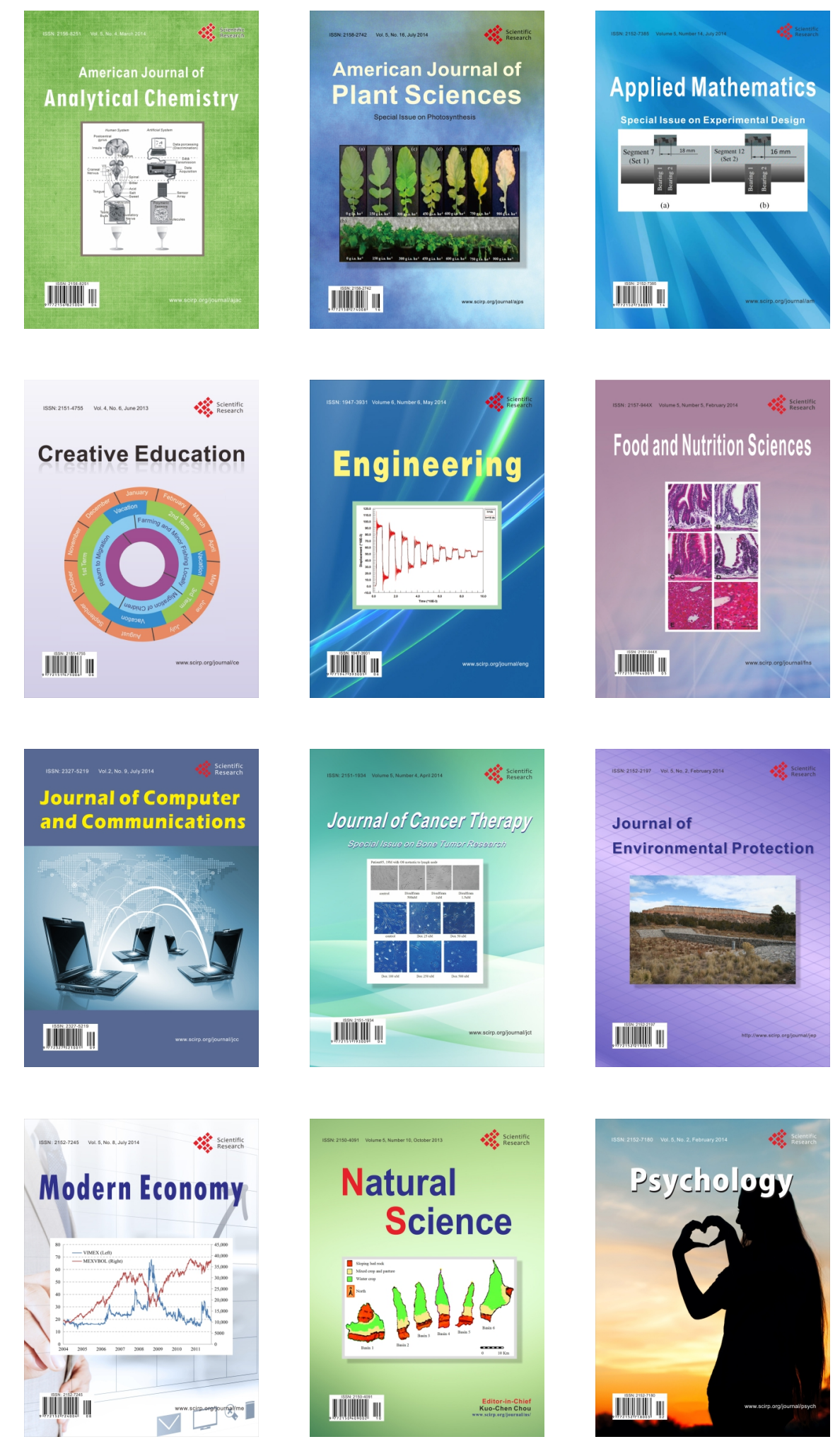